XI.

Ans dem Pharmakologischen Institut der Universität Berlin.

(Direktor: Prof. Dr. Heffter.)

\title{
Zur Frage über das Schicksal der Salizylsäure und einiger ihrer Derivate im 0rganismus.
}

\author{
Von \\ W. Devrient (Kasan).
}

Es wurde der Frage uiber das Schicksal der Salizylsäure im tierischen Organismus von den Forschern stets ein großes Interesse geschenkt. Und auch mit Recht, denn durch die Lösung dieser Frage schien es einerseits am zweckmäßigsten zur Kenntnis des Abbaues des Benzolringes im Organismus zu gelangen, andererseits aber hat gerade die Lösung dieser Frage die beste Möglichkeit versprochen iiber das Wesen der therapeutischen Wirkung der zyklischen Verbindungen überhaupt eine wahrheitsentsprechende Vorstellung zu gewinnen.

Hier schien zunächst die Frage über die Dauer der Ausscheidung der Salizylsä̈rre und die quantitativen Verhältnisse dabei nicht genügend präzisiert zu sein. Unmittelbar darauf sollte die Behanptung Bronardels ${ }^{1}$ ) über die Unterschiede in der Ausscheidung der Salizylsäure in verschiedenem Lebensalter entweder ihre Bestätigung, oder endgültige Widerlegung finden. Diesen Versuchen sollte die Wahl der dazu geeigneten Methode und ihre Prüfung voransgesehickt werden.

Das Studium der Literatur inber das Verhalten der Salizylsänre im Organismus, welche bereits bis zum Jahre 1915 die Zahl von S. 104.

1) Brouardel, Les empoisonnements criminels et accidentiels. Paris 1802 . 
455 Arbeiten umfaßte und mehrmals kritisch bearbeitet worden ist, hat mich zur Überzeugung gebracht, daB man von den alten $\mathrm{Me}-$ thoden der Bestimmung der Salizylsäure, wie es diejenigen von Mosso, Bondzyński, Bourget u. a. sind, Abstand nehmen müsse, da man kein Vertrauen zu diesen komplizierten Methoden, die auf gravimetrischem Prinzipe stehen, haben kann. Derselbe Schluß hat auch Hanzlik und Thoburn ${ }^{1}$ ) bewogen, ihre eigene Methode, welche auf Destillation und Kolorimetrie beruht, auszuarbeiten. Doch schien mir die 1912 veröffentlichte Methode von Sauerland ${ }^{2}$ ) keine geringeren Vorzuige zu bieten, besonders was die einfache Ausführung anbetrifft, und so hielt ich es für geboten, auf die Arbeiten vor der Veröffentlichung Sauerlands nicht weiter einzugehen, sondern seine Methode nochmals zu prïfen und bei erwiesener Brauchbarkeit mit ihrer Hilfe an die Lösung der gestellten Aufgabe zu schreiten.

Bei meinen Versuchen bin ich in der Handhabung der kolorimetrischen Methodik selbst von der Methode Sauerlands abgewichen. Zunächst hatte ich die Absicht zur Ablesung das Chromophotometer nach Plesch heranzuziehen, da ich mir von der sinnreichen Einrichtung dieses Apparates, hanptsächlich aber von dem dort vorhandenen Lummer-Brodhunschen Würfel, viel versprach. Doch hat sich dieser Apparat als so empfindlich und genau erwiesen, daß man ihn für die in Frage kommenden Zwecke gar nicht benutzen konnte. Daraufhin habe ich ein einfacheres Kolorimeter vorgezogen, welches aus 6 Zylindern mit verschiedenen Konzentrationen der Testlösung and einem verschiebbaren 7. mit der zu untersuchenden Flüssigkeit bestand. Die Zylinder waren an einer Wandung aus mattem Glase angebracht. Es wurde so abgelesen, daB ich jedesmal eine genau abgemessene aufsteigende Skala von bestimmtem Natriumsalizylatgehalt (meist von $0,0001-0,0006$ ) bereitete, die zu untersuchende Flüssigkeit erst in einem größeren Zylinder auf das Notwendige verdiunnte, und dann die genaue Einstellung nach dem Kolorimeter ausführte. Im übrigen wurde genau nach den Angaben Sauerlands verfahren. Es scheint mir dringend geboten, an dieser Stelle darauf hinzuweisen, daß der Harn nicht zu übermäßig stark angesänert werden soll, da ein Überschnß an Säure die Stärke der Farbenreaktion beeinträchtigt. Hanzlik (s. oben) hat eine Abnahme in der Konzentration der Testlösung $(0,1: 1000)$ nach Verlanf einiger Zeit beobachtet, und empfiehlt daher dieser Lösung: Chloroform hin-

1) Journal of Biological Chemistry 1915, vol. XXIII.

2) Biochemische Zeitschrift Bd. 80. 
zuzufügen. Das erscheint mir zweckmäßig, obgleich ich solche Stärkeabnahme der Lösung nach 2 Monaten nicht nachweisen konnte. Jedenfalls bilden sich im Laufe der Zeit beträchtliche Kolonien von Schimmelpilzen, die sich mikroskopisch leicht beobachten lassen. Ob diese Schimmelpilze die Spaltung des Natriumsalizylats und dadurch die Stärkeabnahme der Lösung hervorrufen, muß noch nachgewiesen werden.

\section{Versuche zur Prüfung der Methode nach Sauerland.}

1. $10 \mathrm{ccm}$ Harn, enthaltend $5 \mathrm{mg}$ Natr. salicyl. Kahlbanm $=0,00431 \mathrm{mg}$ Salizylsäure, wurden nach der Methode Sauerland untersucht, doch aber ohne Zusatz von Ammoniumsulfat. Es wurde 5 mal mit dem PetrolătherChloroformgemische extrahiert und 3 mal nachher mit Wasser- und Eisenalaunlösung. Es wurden $75 \%$ Salizylsäure wiedergefunden.

2. $10 \mathrm{ccm}$ Harn, gleichfalls enthaltend $5 \mathrm{mg}$ Natr. salicyl. Kahlbaum, wurden génau nach den Angaben Sauerlands untersucht. Es wurde auch 5 mal mit dem Petroläther-Chloroformgemisch und 3 mal mit Wasserund Eisenalaunlösung ausgeschüttelt und dabei 100\% der Salizylsäure wiedergefunden.

3. $10 \mathrm{ccm}$ Harn, enthaltend $5 \mathrm{mg}$ Natr. salicyl. Kahlbaum, wurden nach der Methode Sauerlands nntersucht. Doch nur 1 mal mit dem Petroläther-Chloroformgemisch und 2 mal mit Wasser- und Eisenalaunlösung ausgeschüttelt. Hierbei wurden $94 \%$ der zugegebenen Salizylsäure gefunden.

In diesen drei Versuchen tritt deutlich zutage, daß die Methode Sauerlands zuverlässige Resultate liefert, und daß eine einmalige Petroläther-Chloroformansschïttelung dieses Resultat sichert. Durch die.einfache Ausführung zeichnet sich diese Methode gegenüber den anderen vorteilhaft aus. In Wirklichkeit habe ich mehrfache und verschiedenartig gestaltete Versuche ausgeführt, doch der Kürze wegen teile oben nur die drei notwendigsten mit. Die Lösungen wurden von Herrn Dr. Zondek, Assistenten am Institat, zubereitet; ihre Stärke wurde mir erst nach meiner Mitteilung tuber den verlaufenen Versuch kundgegeben und die Übereinstimmung bestätigt. Nachdem die Methode auf oben geschilderte Weise geprüft worden war, bin ich zu den Versuchen, welche den $Z_{\text {weck }}$ dieser Arbeit ausmachten, geschritten. Es mußten die Ausscheidungsverhältnisse der Salizylsäure beim gesunden Menschen, abhängig von dem Lebensalter, studiert werden. Dementsprechend wurde eine Reihe von Versuchen an drei Männern in folgender Weise angestellt: 


\section{Versuch 1.}

Ein gesunder Mann von 32 Jahren hat morgens um $8 \mathrm{Uhr} 1,0 \mathrm{~g}$ Natr. salieyl. Kahlbaum $=0,862 \mathrm{~g}$ Salizylsäure eingenommen.

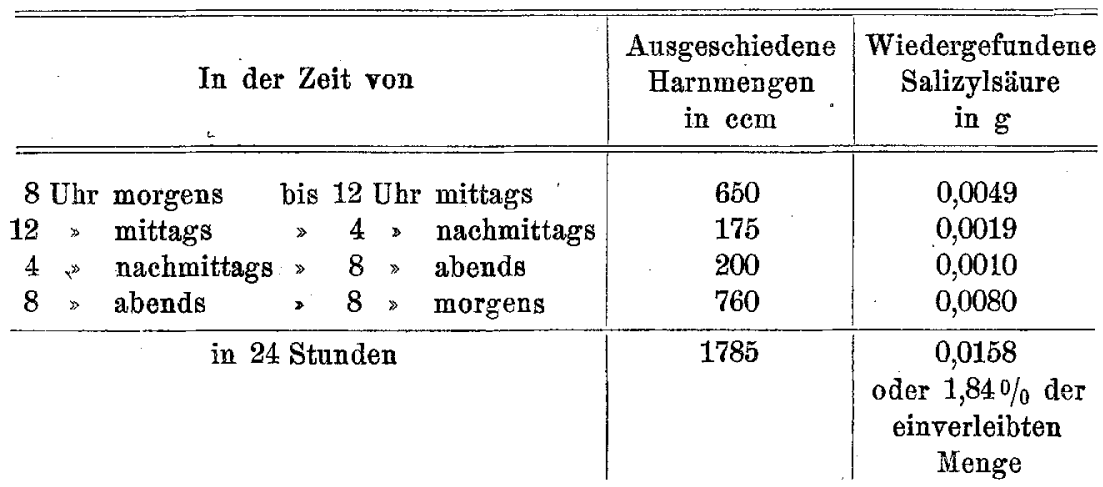

In den Harnausschüttelungen der 7 darauf folgenden Tage konnte keine Salizylsäure nachgewiesen werden.

Versuch 2 .

Ein gesunder Mann von 27 Jahren. $1 \mathrm{~g}$ Natr. salicyl. $=0,862 \mathrm{~g}$ Salizylsäure.

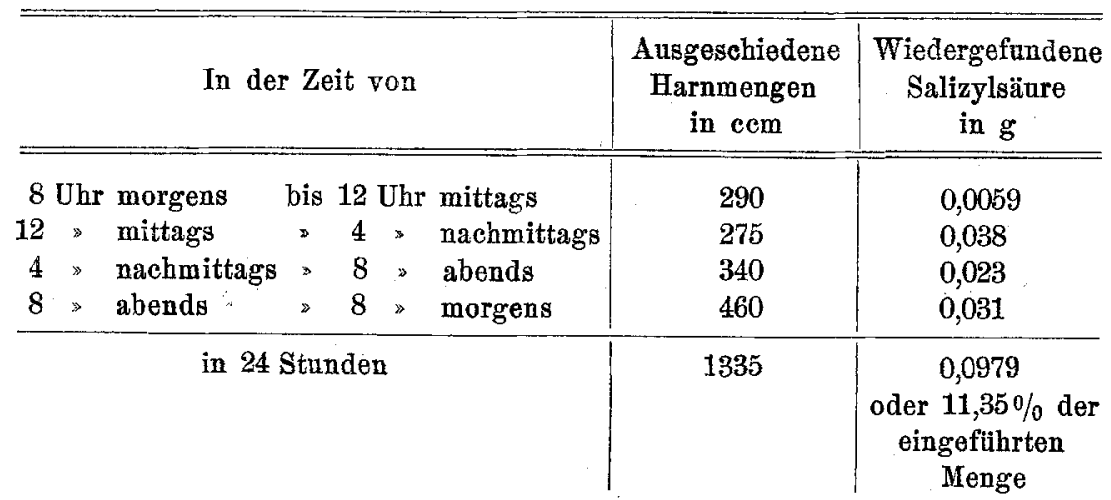

Die Harnausschüttelungen der 7 darauf folgenden Tage zeigen keine Anwesenheit der Salizylsäure an. 
Versuch 3.

Versuchsperson des Versuchs 1. 1,0 g Natr. salicyl. $=0,862 \mathrm{~g}$ Salizylsäure.

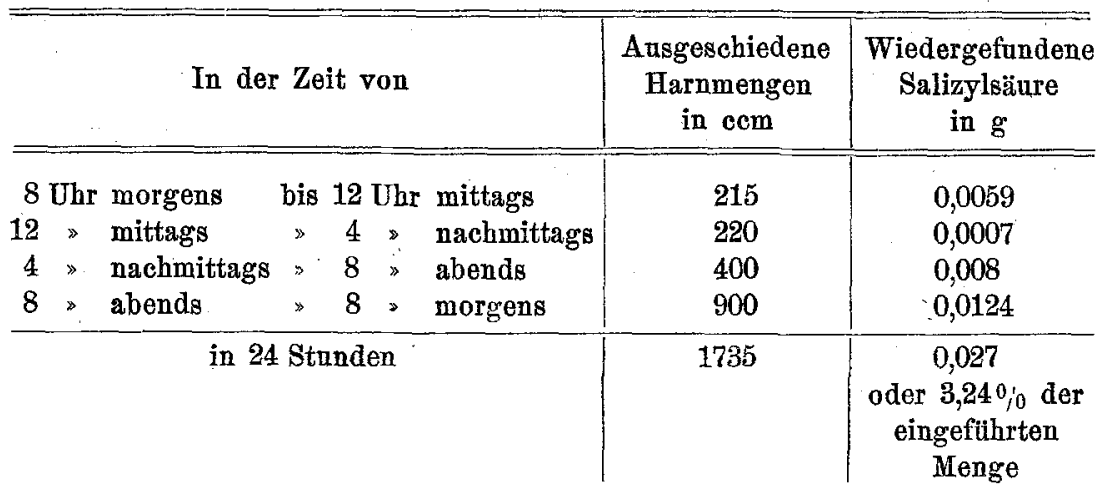

Keine Salizylsäure im Harn der 7 daiauffolgenden Tage.

Versuch 4.

Ein gesunder Mann von 62 Jahren. $1 \mathrm{~g}$ Natr. salicyl. $=0,862 \mathrm{~g}$ Salizylsäure.

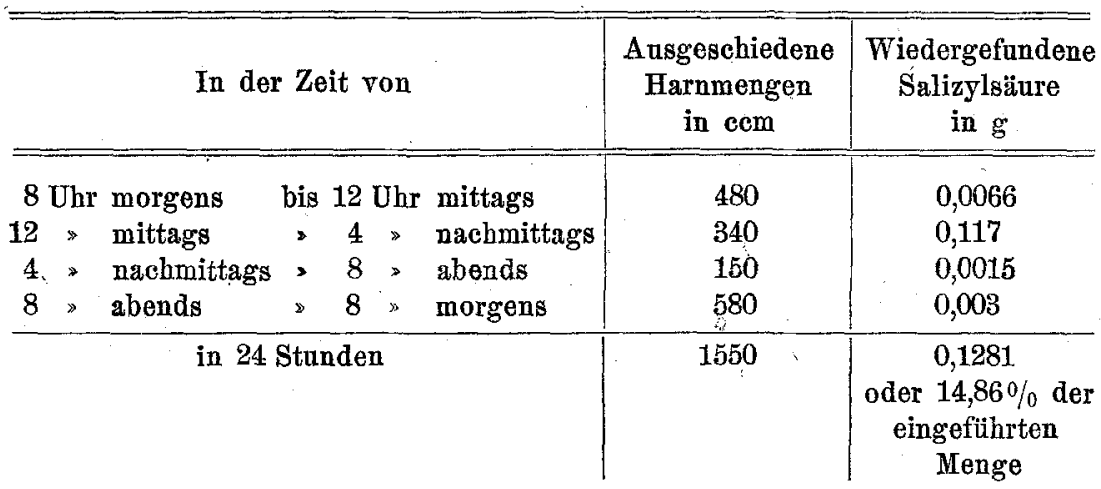

Dieselben Ausschüttelungen sind am darauffolgenden Tage weitergeführt worden. Von 8-12 Uhr war keine Salizylsäure vorhanden, dagegen in der Ausschüttelung von $12-4$ Uhr konnte noch $0,00017 \mathrm{~g}$ Salizylsäure nachgewiesen werden, Die weitere Verfolgung der Auffindung der Salizylsäure bis zum 8. Tage ist ergebnislos geblieben. 
Zar Frage über das Sehicksal der Salizylsäire usw.

Versuch 5 .

Versuchsperson des 1 . Versuchs. $1 \mathrm{~g}$ Natr. salicyl. $=0,862 \mathrm{~g}$ Salizylsäure.

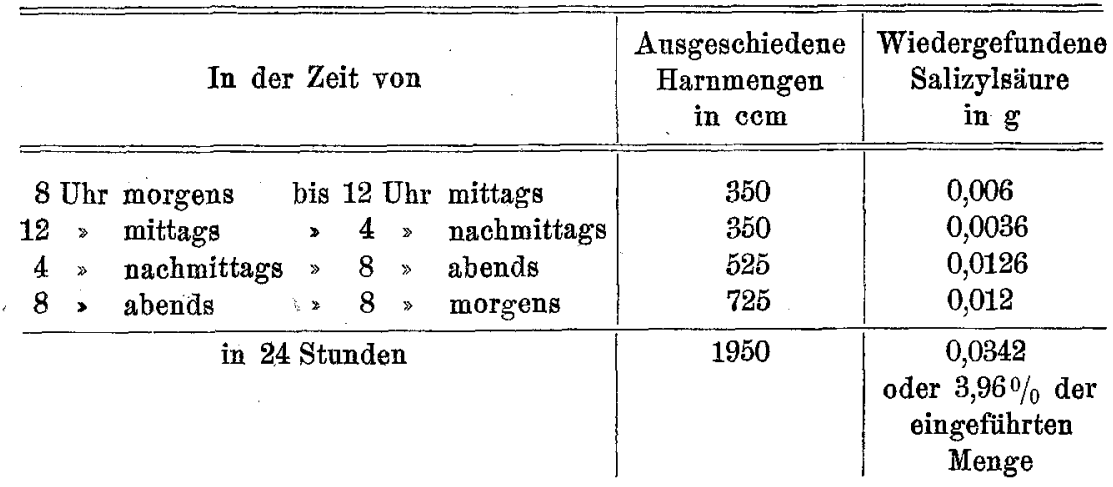

Am 2. Tage:

\begin{tabular}{rl|c|c}
\hline In der Zeit von & $\begin{array}{c}\text { Ausgeschiedene } \\
\text { Harnmengen } \\
\text { in cem }\end{array}$ & $\begin{array}{c}\text { Wiedergefundene } \\
\text { Salizylsüure } \\
\text { in } \mathbf{~}\end{array}$ \\
\hline \hline 8 Uhr morgens & bis 12 Uhr mittags & 400 & 0,0013 \\
$12>$ mittags & $>4 \%$ nachmittags & 340 & $\begin{array}{c}0,0032 \\
\text { unbestimmbare } \\
\text { Spuren }\end{array}$
\end{tabular}

Versuch 6.

Versuchsperson des 2. Versuchs. $1 \mathrm{~g}$ Natr. salicyl. $=0,862 \mathrm{~g}$ Salizylsäure.

\begin{tabular}{|c|c|c|}
\hline In der Zeit von & $\begin{array}{l}\text { Ausgeschiedene } \\
\text { Harnmengen } \\
\text { in ecm }\end{array}$ & $\begin{array}{l}\text { Wiedergefundene } \\
\text { Salizylsäure } \\
\text { in } g\end{array}$ \\
\hline 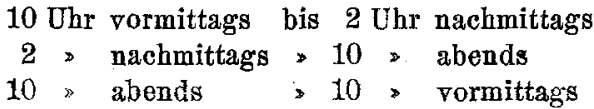 & $\begin{array}{l}230 \\
430 \\
500\end{array}$ & $\begin{array}{l}0,0043 \\
0,0296 \\
0,001\end{array}$ \\
\hline in 24 Stunden & 1160 & $\begin{array}{c}0,0349 \\
\text { oder } 4,05 \% \text { der } \\
\text { eingeführten } \\
\text { Menge }\end{array}$ \\
\hline
\end{tabular}

Keine Salizylsäure in den 7 daranffolgenden Tagen. 
Versuch 7.

Versuchsperson des 4. Versuchs. $1 \mathrm{~g}$ Natr. salicyl. $=0,862 \mathrm{~g}$ Salizylsäure.

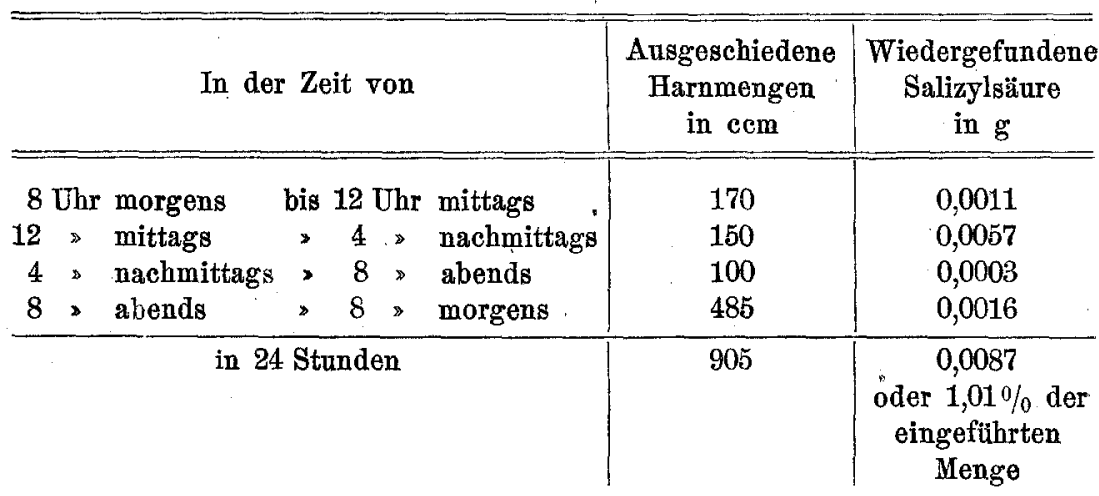

Am 2. Tage:

\begin{tabular}{c|c|c}
\hline In der Zeit von & $\begin{array}{c}\text { Ausgeschiedene } \\
\text { Harnmengen } \\
\text { in ccm }\end{array}$ & $\begin{array}{c}\text { Wiedergefundene } \\
\text { Salizylsäure } \\
\text { in } \mathrm{g}\end{array}$ \\
\hline \hline 8 Uhr morgens bis 12 Uhr mittags & $\begin{array}{c}180 \\
140\end{array}$ & $\begin{array}{c}0,0025 \\
0,0084\end{array}$
\end{tabular}

In den darauf folgenden 6 Tagen keine Salizylsäure vorhanden.

Versuch 8.

Versuchsperson des 1. Versuchs. 1,0 g Azetylsalizylsäure Heyden per os $=0,821 \mathrm{~g}$ Salizylsäure.

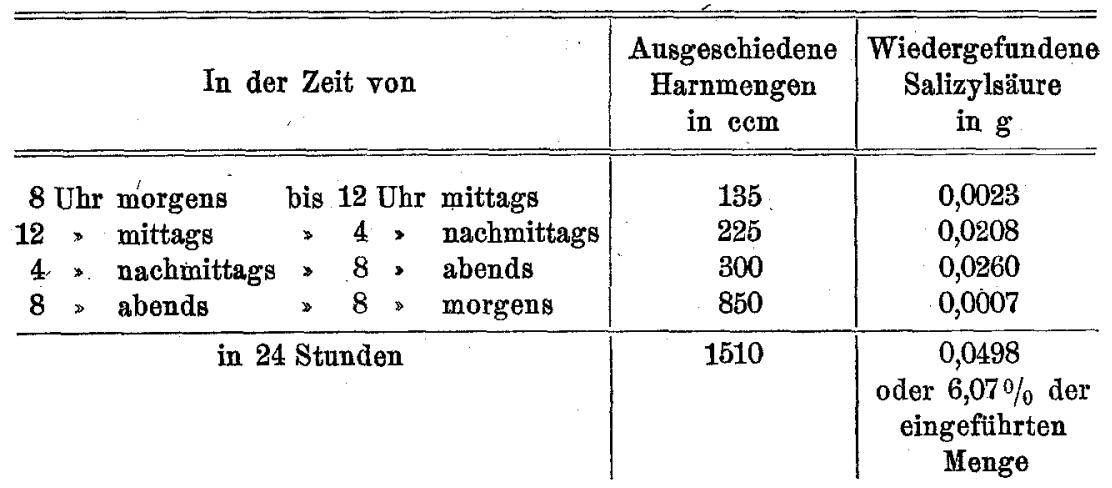

Geringste Spuren in den Aússchüttelungen des 2. Tages. Keine Salizylsäure von dem 3 . Tage an. 
Zur Frage über das Schicksal der Salizylsäure usw.

Versuch 9.

Versuchsperson des 1. Versuchs. 1,0 g Apyron (lösliches azetylsalizylsaures Magnesium) per os $=0,833 \mathrm{~g}$ Salizylsäure.

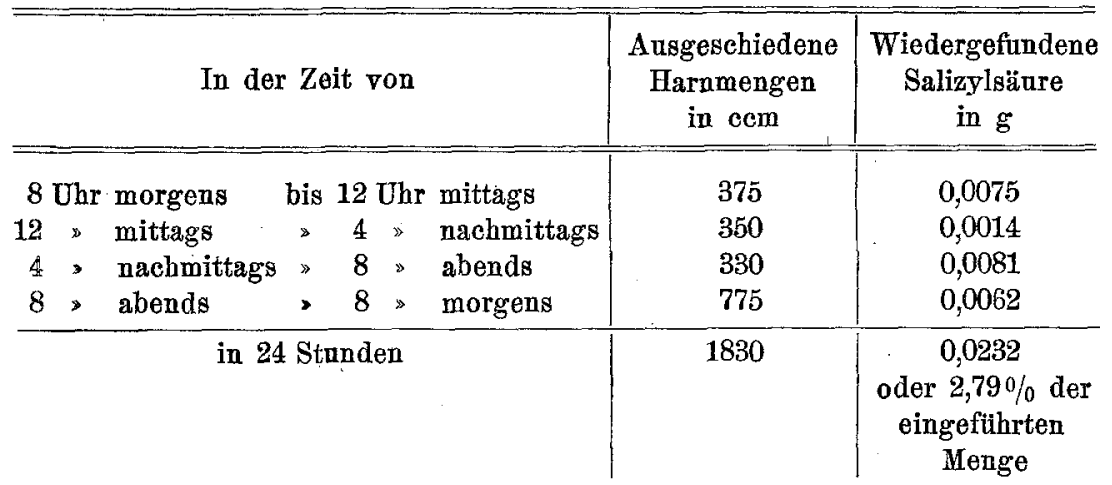

Die Ausschüttelungen des 2. und 3. Tages zeigen Spuren der Salizylsäure an. - Vom 4. bis zum 8. Tage keine Salizylsäure vorhanden.

Bei der Berechnung wurde angenommen, daß die Formel des Apyrons aus 2 Molekeln der Azetylsalizylsäure bestände. Ein zur kolorimetrischen Bestimmung der Salizylsäure im Apyron vorgenommener Versuch mißlang. da dasselbe sich äußerst schwer zu verseifen scheint.

Versuch 10.

Ein gesunder Mann von 31 Jahren hat um 8 Uhr morgens 2,9991 g Äthylester der Salizylsäure $=2,702 \mathrm{~g}$ Salizylsäure eingenommen.

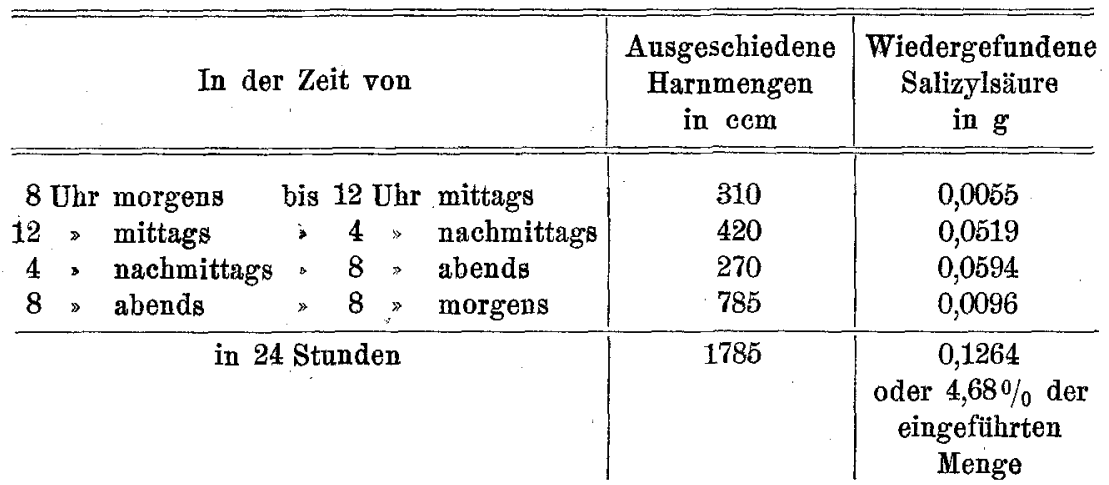

Die Ausscheidung am 2. Tage ergab:

\begin{tabular}{c|c|c}
\hline In der Zeit von & $\begin{array}{c}\text { Ausgeschiedene } \\
\text { Harnmengen } \\
\text { in ccm }\end{array}$ & $\begin{array}{c}\text { Wiedergefundene } \\
\text { Salizylsäure } \\
\text { in g }\end{array}$ \\
\hline \hline $\begin{array}{l}8 \text { Uhr morgens bis 12 Uhr mittags } \\
12>\text { mittags } \$ 4 \$ \text { nachmittags }\end{array}$ & $\begin{array}{c}270 \\
325\end{array}$ & 0,0008 \\
0,0006
\end{tabular}


In den darauffolgenden Ausschüttelungen bis zum Ende des 3. Tages geringe Spuren der Salizylsäure vorhanden. Vom 4. bis zum 8. Tage ist keine Salizylsäure gefunden worden.

Die hier angegebene Quantität des Äthylesters der Salizylsäure wurde analog dem Versuche von Bondzyński (Arch. f. exper. Pathol. u. Pharm. Bd. 38) gewählt, welcher nach seiner gravimetrischen Methode dabei 91,3\% der Salizylsäure im Harn wiedergefunden hat.

Brouardel (s. oben) hat während einer Mahlzeit drei Personen za je $1,0 \mathrm{~g}$ Salizylsäure gelöst in $1 / 21$ Wein gegeben und die Ausscheidung dabei verfolgt. Bei der ersten Person im Alter von 23 Jahren erschien die Salizylsäure nach $1 / 4$ Stunde im Harn und die gänzliche Ausscheidung vollzog sich in 24 Stunden. Bei der zweiten Person im Alter von 46 Jahren; begann die Ausscheidung durch den Harn 2 Stunden nach der Einverleibung und dauerte 48 Stunden. Endlich erschien bei der dritten Person im Alter von 68 Jahren die Salizylsäure im Harn erst nach 48 Stunden, um 8 Tage lang zu dauern.

Wie aus meinen Versuchsprotokollen zu ersehen ist, liegen die tatsächlichen Verhältnisse ganz anders. Die Salizylsäure wird unabhängig vom Alter bei gesunden Menschen in Mengen, die nicht 14,86\% der eingeführten Menge übersteigen, zur Ausscheidung gebracht. Die Ausscheidung vollzieht sich unabhängig vom Alter binnen der ersten 24 Stunden nach der Einverleibung, tritt selten in Spuren am 2. bzw. 3. Tage auf, und kann nie an den 5 noch darauffolgenden Tagen gefunden werden. Nicht das Lebensalter, sondern die allgemeinen Abbauverhältnisse scheinen dabei eine Rolle zu spielen. Hier befinden sich meine Versuche in prinzipiellem Einklang mit denjenigen Hanzliks ${ }^{1}$ ), welcher vermittels seiner Methode $79,9 \%$ der eingeführten Salizylsäuremenge im Harn wiedergefunden hat, und daher annimmt, daß nur ungefähr $20 \%$ der einverleibten Salizylsäure im Organismus des gesunden Menschen zerstört wird. Die Zerstörbarkeit nimmt nach seinen Beobachtungen bei Fịeberzuständen und Based ow scher Krankheit beträchtlich za. Somit sind die früheren Behauptungen von $\mathrm{Mosso}^{2}$ ), Bondzyński ${ }^{3}$ ) u. a. über die vollständige Auffindung der eingeführten Salizylsäure im Harn und die daraus folgende Annahme uber ihre Unzerstörbarkeit im Organismus

1) Hanzlik and Thoburn, 7. Biol. Chem. 1916, Bd. 23, S. 163. - Hanzlik, Scott and Thoburn, J. of Pharmacol. and exp. Ther. Bd. 9, 10 und 14.

- Hanzlik and Wetzel, Ebenda Bd. 14.

2) Mosso, Arch. f. exper. Pathol. u. Pharm. Bd. 26.

3) Bondzyíski, Ebenda Bd. 38. 
widerlegt. Meine Resultate differieren zwar in quantitativer Beziehung mit denjenigen Hanzliks, doch ändert das nichts an der These der Zerstörbarkeit der Salizylsäure im Organismus, über welche wir einig sind. In der Tat, wenn die Salizylsäure nicht gänzlich zur Ausscheidung gelangt, so kann sie entweder abgebaut oder anfgespeichert werden. Wie sich aus den Versuchen mit dem Harn und noch folgenden eindentigen Versuchen mit Organen ergibt, sind meine Resultate als Beweise für die Zerstörbarkeit der Salizylsäure im Organismus aufzufassen und mïssen den Respltaten Hanzliks und seiner Mitarbeiter an die Seite gestellt werden.

$\mathrm{Zu}$ äbnlichen Schlußfolgerungen gelangt auch Friedrichsen 1 , dessen Arbeit uiber die quantitative Bestimmung der Salizylsäure im Blute und Harn der Kaninchen ich großen Wert beimesse. Er hat gezeigt, daß nur eine uinbedeutende Menge der injizierten Salizylsäure mit dem Harn während der Versuche ansgeschieden wurde, und daß der überwiegende Teil der injizierten Salizylsäure bald das Blut verläßt, um in die Gewebe einzudringen. Über das unbedentende Auftreten der Salizylsäure in Fäzes und Schweiß ist man ja längst schlüssig geworden (Bondzyński, Hanzlik).

Da mir infolgedessen und auf Grund eigener Erfahrungen die Verhältnisse im Blute und Harn genügend geklärt erschienen, so unternahm ich weiter einige Versuche zum Studium des Verhaltens der Salizylsäure in den Geweben selbst. Doch stellten sich hier Hindernisse in den Weg, welche meine langwierigen Versuche nur zu einer vergleichenden Skizze ausgestaltet haben. Es mißlang der Versuch, eine einwandfreie Methode der Gewinnung der Salizylsäure aus den Organen anszuarbeiten, ein Mißgeschick, welches auch Hanzlik and seinen Mitarbeitern zuteil wurde. Ich kann meine Ergebnisse den umfangreichen Versuchen Hanzliks an die Seite stellen, welche die Zerstörbarkeit der Salizylsäure in den Geweben wohl nachweisen, aber doch vor der Unmöglichkeit der Wiedergewinnung der Salizylsäure aus den Geweben haltmachen. Dixon ${ }^{2}$ ) betont die Möglichkeit einer Spaltung der Salizylsäure durch die Leber, was auch von Hanzlik hervorgehoben wird. Dieser Voraussetznng einer Mißstörung der Molekel der Salizylsäure im lebenden Gewebe will ich mich gerne anschließen, doch bei Versuchen mit toten Organen muß auch an eine Adsorption gedacht werden. Ich habe mehrfach die Beobachtung machen können, daß einer frischen

1) Friedrichsen, Arch. f. exper. Pathol. u. Pharm. Bd. 80, S. 251.

2) Dixon, Manual of Pharmacology 1915. 
Leber oder Plazenta zugesetzte und sofort der Untersuchung nach der unten beschriebenen Methode unterworfene Salizylsäure sich nicht quantitativ wiederfinden läßt. Da in diesem Falle die Dauer der Einwirkung des frischen Gewebes auf die Salizylsäure sehr kurz ist (einige Minuten), und ihr unmittelbar die Extraktion mit saurem Alkohol folgt, so ist dem Gedanken einer chemischen Umsetzung bzw. Spaltung die Annahme einer Adsorption vorzuziehen. Daher ist anch der von mir angewandten Methode der Wiedergewinnung der Salizylsäure aus den Organen nur eine relative Wertschätzung beizumessen. Ich habe beabsichtigt eine Extraktionsmethode anszuarbeiten und derselben die quantitative Bestimmung nach Sa uerland anzuschließen. Dabei wurde wie folgt verfahren:

Die Organe (frische Leber und Plazenta) wurden zerkleinert, gewogen, mit $10 \mathrm{ccm}$ gesättigter Chloroformlösung durchtränkt, mit ẹiner bestimmten Menge Natriumsalizylat versetzt und bei Zimmertemperatur gut verschlossen stehen gelassen. Nach Verlauf einer bestimmten Zeit wurde das Organ mit einem 10 fachen Quantum schwach angesäuerten $90^{\circ}$ igen Alkohols übergossen, auf dem Wasserbade zum Sieden gebracht, abgegossen und abfiltriert. Dasselbe $5 \mathrm{mal}$ wiederholt. 'Die alkoholischen Filtrate wurden vorsichtig abgedampft und das erhaltene kleine Volumen der jetzt schon wässerigen Lösung abgekühlt und filtriert. In diesem Filtrate wurde die Salizylsäure nach Sauerland bestimmt.

\section{Versuche mit Organen.}

1. $\mathrm{Zu} 40,0 \mathrm{~g}$ Kaninchenleber wurden $0,05 \mathrm{~g}$ Natriumsalizylat $=$ $0,0431 \mathrm{~g}$ Salizylsäure zugesetzt. Nach Verlauf von 20 Stunden wurde. velmittels des oben beschriebenen Verfahrens $16 \%$ der zugefügten Salizylsäuremenge wiedergefunden.

2. 40,0 g Kaninchenleber wurden mit $0,05 \mathrm{~g}$ Natriumsalizylat angesetzt. Nach 88 Stunden wurden $4,5 \%$ der zugesetzten Salizylsäuremenge gefunden.

3. $50 \mathrm{~g}$ Plazenta mit $0,05 \mathrm{~g}$ Natriumsalizylat angesetzt. Nach 40 Stunden $25 \%$ der zugefügten Salizylsäuremenge gefunden.

4. $50,0 \mathrm{~g}$ Plazenta mit $0,05 \mathrm{~g}$ Natriumsalizylat angesetzt. Nach 115 Stunden $2 \%$ der zugesetzten Salizylsäuremenge gefunden.

5. Einem Kaninchen von $1,65 \mathrm{~kg}$. Gewicht wurde $1,0 \mathrm{~g}$ Natriumsalizylat $=0,862 \mathrm{~g}$ Salizylsäure subkutan injiziert. Das Tier wurde nach 24 Stunden getötet und folgende Organe in Arbeit genomenen: Blut $=38,5$; 2 Kniegelenke $=23,5 ; 2$ Nieren $=21,5 ;$ Lunge $=25,5 ;$ Leber $=79,5$ und der binnen 24 Stnnden gesammelte Harn $=33,5$. Im Harn sind 0,017 oder $1,97 \%$ der eingeführten Salizylsäure wiedergefunden worden. In der Leber 0,003 oder $0,35 \%$ der injizierten Menge. In ibrigen Organen ist keine Salizylsäure gefunden worden.

Diese Versuche weisen zunächst nach, daß die Salizylsäure vom toten Gewebe entweder gespalten, oder adsorbiert wird. Eher das 
letztere. Um diese Tatsache noch weiter zu verfolgen, habe ich eine $5 \%$ ige Gelatineaufquellung in gesättigter Chloroformlösung mit $0,05 \mathrm{~g}$ Natriumsalizylat 24 Stunden lang bei $15^{\circ}$ stehen gelassen' und nach der oben beschriebenen Methode untersucht. Es konnten nur 44\% der zugesetzten Salizylsäuremenge nachgewiesen werden. Ein gleichzeitig unternommener Kontrollversuch ergab $39 \%$ der zugesetzten Menge. Auch hier liegen, scheinbar, Adsorptionsprozesse vor. Der negative Befund der Salizylsäure in den Organen, außer der Leber, beim Injektionsversuch kann die Voraussetzung über die Zerstörbarkeit der Salizylsäure im tierischen Organismus unterstützen. Obgleich infolge der Adsorption der Salizylsäure durch das Gewebe die Methodik nicht als einwandfrei betrachtet werden $\mathrm{muB}$, so ist doch der glatt negative Befund in den Organen, mit Ausnahme der Leber, immerhin kennzeichnend, da die nicht adsorbierte Menge, falls sie dort vorhanden wäre, jedenfalls sich doch bestimmen ließe. Andererseits aber, wenn die Adsorption in dem Maße vor sich gehen sollte, daß auch die Spuren verschwinden, was durch andere Versuche nicht bestätigt wird, so wird die Grenze zwischen einer Adsorption und einer Spaltung unbestimmbar. Gerade die Extraktion aus den Organen bereitet diese Schwierigkeiten, auf welche seinerzeit schon Bondi und Jacobyl) hingewiesen haben.

Die darauf folgende kolorimetrische Bestimmung in dem von Lipoiden und Eiweißstoffen befreiten wässerigen Filtrate geht zuverlässigerweise vonstatten. Es sei gerade hier noch erwähnt, daß in letzter Zeit Ciamician und Ravenna ${ }^{2}$ ) darauf hingewiesen haben, daB die Salizylsäure, abgesehen von der partiellen Oxydation, in Verbindungen übergeht, welche nicht leicht zu extrahieren sind.

Es können hier solche Verhältnisse vorliegen und weiteres Forschen ist hier geboten.

\section{Versuche über die Ausscheidnng des Aspirins im Harn.}

Neuerdings hat Pitini ${ }^{9}$ ) Versuche ïber die Ausscheidung des unveränderten Aspirins im Harne der Hunde veröffentlicht. Es schien mir erwünscht, anschließend an meine Versuche über das Verhalten der Salizylsäure, diese Beobachtung Pitinis anch an Menschen zu prüfen. Zu diesem Zwecke wurden 2,0 g Azetylsalizylsäure Heyden

1) Bondi und Jacoby, Hofmeisters Beiträge zar chem. Physiol. u. Pathol. 1906, Bd. 7.

2) Ciamisian und Ravenna, Gaza. chim. ital. 1920, Bd.50.

3) Pitini, Arch. Farmacol. sperim. Bd. 29, S. 113-118. Zitiert nach Chem. Zentrbl. 1920, Bd. III, S. 803. 
(geprüft auf Abwesenheit der Salizylsäare nach D. A. B. V.) gesunden Menschen gegeben und der binnen 12 Stunden ausgeschiedene Harn in der Weise untersucht, daß zwei Portionen zu $25 \mathrm{~cm}$ schwach angesänert wurden, mit Ammoniumsulfat gesättigt und mit doppeltem Volumen Äther kräftig $1 / 2$ Stunde lang ausgeschüttelt. Die Ätherausziige wurden vorsichtig auf dem elektrischen "Wasserbade eingedampft und der Ruickstand in Wasser aufgenommen. Das letzte hat sofort nach dem Versehwinden des Äthers zu erfolgen. In der einen Portion wurde sogleich eine kolorimetrische Bestimmung der Salizylsäure, wie oben beschrieben, unternommen. Die andere Portion wurde durch 1 stïndiges Erwärmen bei $50^{\circ}$ mit etwas Natronlauge verseift, dann mit Salzsäure leicht angesäuert und auch kolorimetrisch untersucht.

Versuch 1.

Ein gesunder Mann von 31 Jahiren hat 2,0 g Azetylsalizylsäure Heyden $=1,64 \mathrm{~g}$ Salizylsäure eingenommen. Der binnen 12 Stunden gesammelte Harn enthielt in $710 \mathrm{ccm}$ :

$$
\begin{array}{ll}
\text { mit Verseifung } & 0,056 \mathrm{~g} \text { Salizylsänre } \\
\text { ohne * } & 0,019 \text { " }
\end{array}
$$

Die Differenz von $0,037 \mathrm{~g}$ Salizylsäure entspricht $0,045 \mathrm{~g}$ Azetylsalizylsäure oder 2,25\% der eingeführten Menge.

\section{Versuch 2.}

Ein gesunder Mann von 27 Jahren hat $2,0 \mathrm{~g}$ Azetylsalizylsäure eingenommen. Der binnen 12 Stunden gesammelte Harn enthielt in $320 \mathrm{cem}$ :

$$
\begin{array}{ll}
\text { mit Verseifung } & \begin{array}{l}
0,0788 \mathrm{~g} \text { Salizylsänre } \\
\text { ohne }
\end{array} \\
0,02 *
\end{array}
$$

Die Differenz von $0,0588 \mathrm{~g}$ Salizylsäure entspricht $0,071 \mathrm{~g}$ Azetylsalizylsäure oder 3,55\% der eingeführten Menge.

Die regelmäßig intensiv auftretende Färbung der verseiften Lösung fand in diesen Versuchen ihre quantitative Bestimmung. Die verseifte Lösung enthält also anßer der direkt ausgesehiedenen Salizylsäure noch Salizylsäure, die aus unverändert abgeschiedenem Aspirin stammt. Die ausgeschiedenen Mengen sind geringer als die, welche Pitini bei Hunden beobachtet hat, aber die Tatsache der Ausscheidung ist somit bewiesen. Also beruht das Verhalten des Aspirins im Organismus nur zum Teil auf dem Salolprinzip: ein Teil wird ohne vorherige Abspaltung der Azetylgruppe resorbiert und unverändert ausgeschieden. 


\section{Zusammenfassung.}

1. Die Methode der quantitativen Bestimmung der Salizylsäure im Harn nach Sauerland wurde geprüft und für genau und zuverlässig gefunden.

2. Es werden die Behauptungen Brouardels über die Unterschiede in der Ausscheidnng der Salizylsäure dureh den Harn in verschiedenem Lebensalter widerlegt: bei jungen sowie bei alten gesunden Menschen wird die Salizylsäure in gleicher Weise ausgeschieden.

3. Es wird nachgewiesen, daB im Gegensatz zu den früheren Ansichten über die vollständige Ausscheidung der Salizylsäure durch den Harn dieselbe nur in Quantitäten, welche $14,86 \%$ der eingeführten Menge nicht übersteigen, zur Ausscheidung gebracht wird.

4. Es werden ferner die Beobachtangen Brouardels über die Dauer der Ausscheidung der Salizylsäure durch den Harn in verschiedenem Lebensalter widerlegt: bei jungen sowie bei alten Menschen wird die Salizylsäure in den ersten 24 Stunden nach der Einverleibung ausgeschieden; die Ausscheidung dehnt sich selten in ganz geringen Quantitäten anf den 2. bzw. 3. Tag hinaus.

5. Es wurden Versuche angestellt, welche als weitere Beweise für die Adsorption bzw. Spaltung der Salizylsäure durch totes Gewebe gelten dürfen.

6. Es wird ein beweisführendes Experiment für die Zerstörbarkeit der Salizylsäure im tierischen Organismus erbracht.

7. Es wird nachgewiesen, daB das Aspirin durch den Harn des Menschen teilweise als Salizylsäure, teilweise unverändert ausgeschieden wird. 